\title{
La voix des " indigènes », ou comment rendre audible des voix rendues muettes. Sociolinguistique dans les archives coloniales
}

\author{
Cécile Van den Avenne \\ Université Sorbonne Nouvelle \\ Paris
}

Eh! Cher ami, vous disais-je à tout moment, vos Noirs s'expriment en vérité comme des académiciens ! - Ma foi oui, me répondiez-vous. Mais que voulez-vous que j'y fasse? Je vous traduis mot pour mot ce qu'ils disent. Si leurs langues sont souples et riches, et capables de rendre des nuances très subtiles, cela témoigne simplement que ces gens de l'Afrique Occidentale ne sont pas du tout les brutes qu'une médiocre littérature coloniale se plaît à représenter. Un beau langage est un chef d'œuvre collectif et inconscient. Ces Noirs ne parleraient pas ainsi s'il n'y avait derrière eux une civilisation très simple, mais une civilisation tout de même...

— Jérôme Tharaud et Jean Tharaud, La randonnée de Samba Diouf, Dédicace à André Demaison

Ce court dialogue met en scène un échange entre André Demaison, administrateur colonial, folkloriste, écrivain, et les frères Tharaud, romanciers français à succès de l'entre-deux-guerre. Si la position affichée se veut humaniste et attentive aux cultures africaines, elle n'en reste pas moins surplombante, et quelque peu condescendante. Les Africains y sont objets d'un discours occidental, ils sont parlés plutôt qu'ils ne parlent, et l'écrit en français recouvre leurs langues, et les fait disparaître.

L'un des enjeux historiographiques contemporains en histoire coloniale est, à rebours d'une histoire écrite " du point de vue des vainqueurs ", de tâcher de produire une " histoire à parts égales " (Bertrand, 2011) qui puisse faire entendre la voix des colonisés au même titre ou tout autant que celles des colonisateurs. C'est bien souvent difficile, parce que les sources dont nous disposons sont des documents qui sont majoritairement le produit des institutions administratives coloniales, ou de scripteurs européens, qu'ils n'offrent de ce fait qu'un seul point de vue, où les voix des colonisés sont bien souvent rendues muettes. Et c'est particulièrement le cas en Afrique sub-saharienne, sur laquelle je travaille, où le médium écrit était quasi exclusivement utilisé par l'appareil colonial, dans un contexte où localement les recours et les maîtrises de la littératie étaient faibles ou le fait d'une toute petite catégorie de la population (élite traditionnelle ou élite intermédiaire produite par le système colonial). L'une des façons de contourner le point de vue unique construit par les archives coloniales a pu être, à un moment de l'historiographie de l'Afrique, d'avoir recours à l'histoire orale, en produisant dès lors d'autres archives, alternatives, à partir d'enregistrements de récits transmis oralement par des locuteurs africains. Cela permet ainsi d'accéder à des événements passés à partir d'un récit transmis au présent par un locuteur qui en est dépositaire. Une autre façon est de lire les textes écrits par des Européens à la recherche de voix africaines, en étant sensible, et cela fait partie de l'analyse, à la façon dont ces voix sont médiatisées. Une troisième façon est de s'intéresser aux textes produits notamment par ces élites intermédiaires, utilisant les ressources de la littératie en langue européenne, à leur propre usage.

Mes recherches portent sur les pratiques langagières qui caractérisent la situation de contact colonial en Afrique de l'Ouest entre le milieu du XIXème siècle et l'après Deuxième Guerre mondiale, c'est à dire la période qui correspond à la colonisation par la France d'une large portion de ce territoire (Van den Avenne, 2017a). Les sciences sociales du langage, au sein desquelles j'inscris mes recherches, et leur attention à la granularité des discours permettent de mettre au jour des processus qui échappent à des lectures davantage orientées vers les faits que vers les mises en mots. II s'agit notamment de rendre compte des enjeux de pouvoir perceptibles dans certaines mises en texte, la façon dont ces textes encodent des inégalités sociales au sens large (englobant notamment la question raciale), cela à travers une analyse fine de leurs différentes composantes matérielles et langagières. Ainsi, au-delà d'un changement de point de vue (faire entendre le point de vue des dominés contre le point de vue unique des dominants) - ce que propose notamment certains historiens, mais également les chercheurs en études subalternes, il s'agit de mettre au jour les mécanismes langagiers qui fabriquent la domination. L'enjeu est évidemment double, à la fois scientifique et politique.

Dans cette contribution, après un cadrage épistémologique et méthodologique, je vous propose d'exemplifier ma démarche à partir de l'analyse de différents documents produits dans un même contexte, celui de la Première Guerre mondiale, première guerre européenne où des soldats originaires des colonies 
Van den Avenne, Cécile. "La voix des « indigènes », ou comment rendre audible des voix rendues muettes. Sociolinguistique dans les archives coloniales. » Nouvelle Revue Synergies Canada, №15

ont été engagés, et moment de l'histoire qui a amplifié les contacts entre Européens et Africains de façon inédite.

II s'agira à partir de ces analyses de documents de révéler deux processus : celui, d'une part, qui a pu rendre inaudible la parole « indigène » dans les documents, les archives produites par des acteurs européens ; et la manière, en retour, dont certains acteurs africains ont tâché de regagner en agentivité en rendant audible leur voix.

\section{Des voix dans les archives - comment les entendre ?}

\section{1 « Pour une compréhension de la voix ». Éléments de définition ${ }^{1}$}

En préambule, j'aimerais tâcher de cerner ce qu'on peut entendre par « voix » dans la perspective sociolinguistique qui est la mienne, en fort dialogue avec ce qui se pratique en histoire. Hymes (1996), dans son ouvrage liminal sous-titré Towards an Understanding Of Voice, sans réellement proposer une définition et une théorisation précise de ce qu'il appelle la notion de voix [the notion of voice] en énonçait cependant quelques caractéristiques, qu'il est intéressant de reprendre. Tout d'abord, il associe « voix » et « liberté » (Hymes, 1996, p. 64), précisant que deux sortes de libertés sont réunies sous la notion de voix : une liberté qu'il dit négative qui viendrait d'une absence d'obstacle linguistique dans la prise de parole, ou dans les actes de littératie, une liberté permise donc par la maîtrise de la langue comme ressource, mais qui est associée à une liberté positive, consistant à pouvoir se faire entendre, à avoir une voix qui porte, qui vaut la peine d'être entendue [ " freedom to have one's voice heard, freedom to develop a voice worth hearing " (Hymes, 1996, p.64)]. En cela, la façon dont Hymes (1996) utilise la notion de voix dépasse par exemple la définition qui en est donnée dans le manuel d'anthropologie linguistique dirigé par Duranti (2007): « the linguistic construction of a social personae » répondant à la question « Who is speaking ? " [ Qui parle ? »] (Keane, 2007, p. 268), faisant de la voix la projection d'une identité sociale, à travers des ressources linguistiques mobilisées. Hymes (1996) y ajoute ce que l'on pourrait décrire comme un élément perlocutoire : la voix est efficace, elle porte, elle se fait entendre. Blommaert (2008b), quant à lui, mobilise cette notion à différentes reprises dans ses travaux, dans la locution « to have voice " [avoir une voix] : "People use language and other semiotic means in attempts to have voice, to make themselves understood by others. » ajoutant « [...] having voice is therefore an intrinsically social process - that is a process with clear connections to social structure: history, culture, power. » (p. 427). Dès lors, les individus se retrouvent dans des positions d'inégalité en termes de possibilité de faire entendre leur voix : «It is clear that an illiterate person has different potential from a literate person ; that a multilingual person has different potential from a monolingual one ; that someone who speaks the prestige variety of a language has different potential from someone who speaks a stigmatized and marginalized variety of a language. » (Blommaert, $2008 b$, p. 428). Les institutions, et plus particulièrement le système éducatif, ont tendance, poursuit Blommaert, à "geler » les voix. II s'intéresse ainsi plus particulièrement à ce qu'il appelle des voix vulnérables : immigrants qui perdent leur voix face au service de police ; ou, à l'inverse, des voix qui peuvent se donner à entendre, alors qu'elles étaient par le passé inaudibles, par exemple celles des témoins au sein de la Commission Vérité et Réconciliation mise en place en Afrique du Sud après l'Apartheid.

Ainsi la voix, telle que travaillée par l'anthropologie linguistique, se rapproche de la notion mobilisée par l'économiste A.O. Hirschmann (1995) et reprise par Payet et al. (2008) dans leur ouvrage La voix des acteurs faibles, où voix signifie " prise de parole » et est une forme de mobilisation sociale et politique (opposée notamment à la défection, " exit »). La rencontre des deux termes, "voix », et " acteurs faibles » (désignant des acteurs disqualifiés, que ce soit au regard d'un principe éthique de dignité, de décence ou de reconnaissance (Payet et al., 2008, p. 9), i.e. aussi bien Sans Domicile Fixe, prostitué(e)s, qu'ouvriers non qualifiés, etc.), souligne à quel point la notion de voix peut être mobilisée pour des voix peu audibles ou généralement peu efficaces et se relie aussi à une autre notion qui est celle d'agentivité [agency]. Des liens forts existent, entre cette notion d'une part et les études coloniales et postcoloniales et d'autre part les études sur les intermédiaires. Ainsi, on rappellera tout d'abord que les penseurs du courant dit des subaltern studies ont été les premiers, au milieu des années 1980, à pointer la nécessité de redonner voix aux « sans-voix » de l'histoire coloniale. La référence ici est bien sûr le texte fondateur de Spivak (1988/2006), « Can the Subaltern Speak ? ». Ces penseurs ont opéré un retour historiographique critique visant à déconstruire « l'archive coloniale » en montrant les contraintes discursives et idéologiques qui ont présidé à la fabrication de telles archives puis formaté leurs usages dans les premiers travaux d'histoire coloniale. 
Van den Avenne, Cécile. "La voix des « indigènes », ou comment rendre audible des voix rendues muettes. Sociolinguistique dans les archives coloniales. » Nouvelle Revue Synergies Canada, №15

La voix est une notion que mobilisent également les historiens, qui s'attachent, dans la lecture et l'analyse des archives, à faire surgir des voix, oubliées, peu audibles, en marge du pouvoir. Un texte fondateur est celui de Certeau (1975) évoquant les « documents altérés » recouvrant les voix réelles des possédées, à laquelle l'historien, s'intéressant à des phénomènes collectifs de possessions et d'exorcisme au début du XVIlème siècle en France, ne peut pas avoir accès. Les sources disponibles (procès-verbaux de notaires, rapports de médecins, avis de théologiens, etc.), précise de Certeau (1975), «présentent souvent le 'discours' de la possédée comme ce qui est toujours énoncé par quelqu'un d'autre » (p. 295). Ces travaux s'inscrivent dans ce que l'on a pris l'habitude d'appeler la micro-histoire (microstoria), prônant la réduction d'échelle et suivant des destinées d'individus d'importance mineure, ayant laissé peu de traces dans les archives écrites.

\subsection{Des voix médiatisées par l'écrit}

Dans un travail sur les archives judiciaires égyptiennes, Clément (2012) présente une analyse fine et stimulante permettant de faire surgir les voix de paysans égyptiens, accusés de crime puis condamnés à mort par les tribunaux nationaux. Elle met en jeu ce qu'elle appelle une " archéologie de la performance », qui permet, à travers les sources écrites (en l'occurrence le dossier judiciaire), de retrouver la performance orale et les interactions qui les sous-tendent. En confrontant deux types d'archives, les dossiers judiciaires (qui traduisent en termes juridiques des prises de parole produites dans un cadre très contraignant), et le « vrac » de lettres et pétitions en annexe des dossiers (révélant des capacités d'appropriation de contestation au sein du système), elle montre ainsi comment il est possible pour l'historien de faire entendre les voix de ces paysans et une forme d'agentivité.

Les travaux de Certeau $(1975,1990)$ ou ceux plus récents de Clément pointent bien la complexité des relations entre oralité et écriture. Dans l'un et l'autre cas, les voix, celles des paysans ou celles des possédées, ne doivent pas « être recherchées dans une sorte d'oralité/altérité sacralisée » mais « dans cet entre-deux qui lie et englobe l'énonciation des paroles [...] et leur transcription sur le papier » (Clément, 2012, p.2). Si la forme dialogique des archives judiciaires permet la mise en œuvre de cette " archéologie de la performance ", d'autres types de corpus ont pu être privilégiés par les chercheurs s'attachant à faire surgir des voix peu entendues, en marge des instances officielles ou des institutions. J'évoquais dans mon introduction la question des archives orales, et je n'y reviendrai pas ici. Un autre type de corpus mobilisable est celui des écrits dits ordinaires.

Ces écrits, en effet, produits souvent par des individus peu lettrés ou du moins n'occupant pas des positions de pouvoir, permettent de faire surgir d'autres voix, en marge des récits et écrits officiels ou dominants. Le terme, " écritures ordinaires ", renvoie aux travaux de Fabre (1993) qui a constitué ces écrits en objets sur des terrains européens, dans les années 1990. Ils divergent notamment des écrits administratifs et des écrits littéraires, fortement codifiés, ce sont des écrits du quotidien, à usage personnel, de genre divers (qui vont de la liste de course, du pense-bête à la correspondance, en passant par le journal intime).

Ces écrits ont été qualifiés de différentes façons : soit à partir des compétences et de la catégorisation des scripteurs selon ces compétences: "semi alphabètes » (semi alfabeti) (Petrucci, 1986), " malhabiles » (Branca-Rosoff, 1989, p. 9), « demi-lettrés » (Branca-Rosoff, 1989, p. 24), soit à partir d'une qualification des textes eux-mêmes : « textes pauvres » (Branca-Rosoff, 1989, p. 5), " grassroots writings » (Blommaert, 2004, 2008a). Dans un cas comme dans l'autre, ces textes sont qualifiés à l'aune de modèles implicites, produits par des lettrés, ayant intégré les normes linguistiques et discursives dominantes. Parler d'écrits ordinaires, comme le fait notamment Fabre (1993), permet d'éviter ce biais, même si sur certains terrains, et c'est particulièrement le cas en Afrique subsaharienne où j'ai par ailleurs mené des terrains contemporains, la prise d'écriture n'est pas si ordinaire que cela.

La perspective se complique quand des textes produits dans une économie locale de l'écrit particulière sont déplacés dans d'autres cercles régis par d'autres normes. Blommaert (2008a), dans ses travaux, s'est ainsi attaché à rendre compte de ce qu'il advient de ces textes lorsqu'ils migrent d'une économie locale de productions et usages à une autre, au sein d'un processus général de globalisation affectant aussi la circulation des textes. II est particulièrement attentif au fait que certains textes, produits par des scripteurs africains en particulier, venant d'environnements culturels et sociaux où les pratiques de l'écrit sont réduites, perdent en efficacité lorsqu'ils sont déplacés dans des réseaux de circulation de l'écrit en occident. II pointe alors ce qui est la tâche du chercheur, linguiste ou anthropologue : reconstruire la valeur de ces textes 
Van den Avenne, Cécile. "La voix des « indigènes », ou comment rendre audible des voix rendues muettes. Sociolinguistique dans les archives coloniales. » Nouvelle Revue Synergies Canada, №15

délogés, déplacés, hétérographiques « dislodged, displaced, hetero-graphic texts » (Blommaert, 2008a, p. 661) (hétérographiques vs. orthographiques). En effet, «la voix du sujet communiquant doit être reconstruite et restaurée, du fait qu'elle ne peut pas, d'elle-même, se faire entendre. ${ }^{2}$ " (Blommaert, 2008a, ibid.) [ma traduction]. Blommaert s'interroge et son interrogation sous-tend aussi mon travail : "Comment construisons-nous des voix africaines à partir de l'analyse de textes écrits africains ? Comment pouvonsnous percevoir l'entièreté d'une voix-comme-savoir [voice-as-knowledge] dans de tels documents ?" (Blommaert, 2008a, p. 662). Et nous ne pouvons nous départir, en posant cette question, d'une prise en compte de notre propre position de chercheuse, blanche, française, issue d'une ancienne puissance impériale et du fait que le colonialisme passé habite nos situations postcoloniales contemporaines.

\section{Des voix rendues inaudibles}

Les voix africaines sont difficiles à faire émerger de la production écrite coloniale. Elles sont soit totalement absentes parce que les textes, écrits par des Européens et depuis le point de vue situé qui est le leur, ne les prend pas en compte, soit parce que différents procédés d'entextualisation ${ }^{3}$ les rendent inaudibles. J'en décrirai ici deux, qui rendent les voix africaines inaudibles dans les productions textuelles coloniales : d'une part la traduction, et d'autre part le figement dans une variété dévaluée de la langue coloniale, en l'occurrence le français. Ces procédés d'entextualisation sont liés à des contraintes de genres discursifs, qu'une socialisation à l'écrit transmet, et dont je fais l'hypothèse qu'elle a en quelques sorte rendus « sourds » les Européens, rendus incapables d'entendre les prises de parole africaine.

L'exclamation qui ouvre le court échange mis en exergue de cet article pointe bien ce phénomène : certaines voix peuvent ne pas être entendues parce qu'elles ne s'expriment pas dans le code attendu, transmis par des habitudes de lecture qui l'ont figé en stéréotype. En l'occurrence, des Noirs s'exprimant « comme des Académiciens » suscitent l'étonnement, parce qu'un stéréotype figé dans la littérature coloniale les fait parler essentiellement en "style télégraphique » pour reprendre une expression de l'administrateur colonial et ethnographe Maurice Delafosse. Parlant ainsi, sont-ils rendus plus audibles que lorsqu'ils sont représentés parlant un français cassé? On peut en fait en douter.

Lorsque Demaison est mis en scène énonçant : « je vous traduis mot pour mot ce qu'ils disent » (op.cit.), il accapare en fait la capacité à faire entendre. La traduction, reposant sur l'illusion d'une forme de transparence, si elle est une façon de donner à entendre, aux Européens, des propos « indigènes », est également une façon d'effacer leurs voix propres. L'effacement est d'autant plus manifeste quand le propos est rapporté, traduit, par un tiers européen. Paradoxalement, dans la littérature coloniale, c'est le « traducteur » qui endosse l'auctorialité des propos. Les indigènes sont parlés, ils ne prennent pas la parole, et cela ne change pas grand-chose lorsque, comme tâche de le faire Demaison, animé par une forme d'humaniste non dénué de paternalisme, on substitue au stéréotype du langage petit-nègre (voir ci-dessous) du français soutenu, cela ne fait que changer la tonalité du texte ${ }^{4}$. Je partirai d'un autre exemple pour illustrer cela. J'ai travaillé de façon précise sur les carnets d'exploration de Louis Gustave Binger, un militaire et explorateur, qui monta une grande mission d'exploration entre 1887 et 1889, qui lui fit parcourir près de 4000 kilomètres entre Bamako au Soudan (actuel Mali) et Grand Bassam, sur la côte de l'actuelle Côte d'Ivoire. Pour documenter cette expédition, nous disposons de dix carnets manuscrits rédigés au fil de l'exploration, ainsi que d'une somme en deux tomes d'environ 900 pages, publiée en 1892 sous le titre $D u$ Niger au Golfe de Guinée, qui est devenu un classique de la littérature d'exploration (pour une étude précise des carnets, je renvoie à mon article, Van den Avenne, 2017b). Je me suis notamment attachée à faire ressurgir, à travers la lecture croisée de ces deux textes très différents, la voix de Diawé Fofana, le compagnon africain principal de Binger durant cette expédition. Je ne donnerai ici qu'un exemple qui va dans le sens de mon argumentation concernant la traduction et l'effacement qu'elle opère. Dans Du Niger au Golfe de Guinée, est relatée une veillée durant laquelle Diawé Fofana raconte à l'assistance une fable en mandingue. Binger l'insère traduite en français dans le récit : « Un jour le lion dit à l'Hyène, [...] » (Binger, 1892, p. 9-12). Dans les carnets de Binger, on trouve une trace de cette veillée de conte, soit la transcription sur trois pages d'un conte en mandingue, écrit au crayon de papier, avec, dans l'interligne, des éléments de traduction et d'élucidation en français, témoignant d'une séance de travail avec Diawé Fofana, dictant et élucidant certaines traductions. La traduction, dans le récit de voyage, donne l'illusion d'un code partagé, et procède d'une forme d'accaparement culturel, le conte devenant partie intégrante du récit de voyage à la première personne, mais et surtout, elle oblitère complètement la dimension de travail derrière cette 
Van den Avenne, Cécile. "La voix des « indigènes », ou comment rendre audible des voix rendues muettes. Sociolinguistique dans les archives coloniales. » Nouvelle Revue Synergies Canada, №15

traduction, travail réalisé de façon conjointe par l'Africain et l'Européen, résultant donc d'une interaction, d'une transaction, dont nous n'avons plus trace dans le texte publié sous le seul nom d'un auteur européen.

De nombreux textes coloniaux, par ailleurs, ont pu rendre inaudibles les voix africaines en les faisant entendre dans une variété de français « cassée », dépréciée, qui historiquement est devenu une iconisation du colonisé africain, le français dit « petit nègre » appelé parfois « français tirailleur » (pour une analyse précise, je renvoie à Van den Avenne, 2019). Cette variété est encapsulée dans des textes aux statuts très divers, et particulièrement dans la littérature de voyage, qui a largement contribué à stabiliser et diffuser ce stéréotype faisant parler les Africains « au mode infinitif » pour reprendre l'expression déjà citée de Maurice Delafosse. L'hypothèse que je fais est que la diffusion de ce stéréotype a pu rendre certains Européens incapables d'écouter et entendre certaines prises de parole africaines, particulièrement lorsqu'elles ne s'expriment pas dans une variété complètement normée de français. J'exemplifie ici à partir d'un seul document (pour une analyse plus approfondie, voir Van den Avenne, 2017a). Il s'agit des notes d'audience d'un conseil de guerre, qui eut lieu le 4 mai 1915 à Sedd-Ul-Bahr ${ }^{5}$ en Turquie, à l'issue duquel six tirailleurs sénégalais incorporés dans le $4^{\mathrm{e}}$ régiment mixte colonial furent reconnus coupables d' « abandon de poste en présence de l'ennemi » et fusillés.

Tidaogo Tampsaba : Pas abandonné mon poste, plus de chef, plus de cartouche moi tout seul chercher bataillon venu jusqu'au village sans trouver

Bada Bara : Moi chercher bataillon, plus de chef-pas vouloir prendre bateau

Katio Veo : Moi bon soldat, bien battu Maroc, en France. pas vouloir partir - pas jeté fusil

Maqui Dioup : Plus capitaine, plus sergent, plus cartouche. venu chercher bataillon - pas vouloir sauver

Baziri arore : pas jeté fusil. c'est gendarme qui l'a pris - pas aller prendre bateau - plus personne venu chercher camarades

Sory Loul baby ${ }^{6}$ : Moi rien mauvais tout bon - pas voulu chercher bateau - pas $X$ fusils $X$ chez Turcs - plus de cartouches - cherché bataillon ${ }^{7}$

Avec les six tirailleurs est également jugé un soldat français, Pierre Liotard, dont les propos sont ainsi transcrits :

Je m'étais battu toute la nuit, un de mes amis est tombé à mes côtés. Une balle a passé devant moi et je n'ai plus vu clair. J'ai eu des convulsions nerveuses. Je suis réformé de la classe 1914 je n'ai pas $X$ je n'ai pas voulu me sauver mais me faire soigner. Je venais d'être mis en réserve et je me rendais à l'hôpital de campagne pour me faire soigner quand j'ai été arrêté. Je demande à retourner en 1ère ligne.

Certes les tirailleurs sénégalais en question parlaient vraisemblablement une variété approximative de français, la plupart ayant appris le français « sur le tas » au sein de l'armée. Le créoliste Robert Chaudenson (2003) décrivait la variété communément appelé dans le corpus colonial " français-tirailleur » comme « pidgin quelque peu artificiel, qui procède à la fois de variétés approximatives produites par des locuteurs en situation exolingue et de généralisations didactiques réputées faciliter et accélérer l'apprentissage de ce français minimal » (Chaudenson, 2003, p. 54) ${ }^{8}$; en même temps, la retranscription, particulièrement laconique, utilise des stéréotypes reconnaissables et on peut faire l'hypothèse que celui en charge de la transcription, parce qu'on est aussi dans le cadre d'une justice militaire expéditive, a retranscrit moins ce qu'il a entendu réellement que ce qu'il pensait entendre, à travers les stéréotypes qu'il avait de la façon dont parlent les Africains. Ainsi, cette retranscription rend la parole des tirailleurs doublement inaudible : d'une part, elle se fige en stéréotype ; d'autre part, elle perd toute efficacité, toute agentivité, dans le cadre contextuel propre de ce tribunal. On ne peut certes en déduire que ces tirailleurs furent fusillés parce qu'on n'a pas su les entendre, mais on peut en émettre l'hypothèse. 
Van den Avenne, Cécile. "La voix des « indigènes », ou comment rendre audible des voix rendues muettes. Sociolinguistique dans les archives coloniales. » Nouvelle Revue Synergies Canada, №15

\section{Regagner en agentivité : faire entendre sa voix dans la langue du colonisateur}

Dans ce deuxième moment, je vais tâcher de rendre compte de la manière dont certains acteurs africains ont tâché de regagner en agentivité en rendant audible leur voix. Regagner en agentivité a d'abord consisté à maîtriser la langue européenne coloniale: s'approprier la langue française en l'occurrence permet éventuellement de produire un contre-discours, de se réapproprier les savoirs produits sur sa propre société, également servir ses intérêts propres et ses ambitions personnelles. La question de la maîtrise de la langue française et de l'écrit en français par les sujets indigènes de l'empire colonial français représente également un enjeu politique, à savoir la possible accession à une citoyenneté française. En France métropolitaine, maîtrise de la langue française et droits politiques sont liés, dans la pratique et l'idéologie, depuis la Révolution française. La mise en place de la scolarisation obligatoire sur le territoire français conduit à renforcer la congruence entre nationalité, citoyenneté et scolarisation en français. Dans les colonies françaises, relevant du territoire national, les populations ont, dans leur très grande majorité et jusqu'en 1956, le statut de sujets indigènes : ils sont ressortissants de l'État français, mais ils sont privés des prérogatives des citoyens. Pour certains membres des élites intermédiaires africaines, l'accession à la citoyenneté française et aux droits qui lui sont associés fut ainsi un enjeu politique important bien avant les revendications d'indépendance.

J'exemplifierai à partir d'un document issu d'un fonds d'archives déposés aux Archives Nationales d'OutreMer (ANOM) et sur lequel je travaille actuellement. II s'agit d'une lettre extraite d'une correspondance envoyée par des tirailleurs dahoméens pendant la Première Guerre mondiale au lieutenant-gouverneur du Dahomey de cette période, Charles Noufflard. Le recrutement des tirailleurs dahoméens lors de ce conflit présente des particularités qui le distingue de ce qui se passa dans le reste de l'empire. En effet, il suscita, parmi les populations dahoméennes, de très fortes oppositions. Au moment de la déclaration de guerre, les seuls à se porter volontaires furent des jeunes gens lettrés, membres de l'élite urbaine locale, et souvent employés dans l'administration coloniale française. Certains demandèrent, à cette occasion, leur citoyenneté française. Charles Noufflard fut chargé d'organiser le recrutement et utilisa comme moyen de propagande des lettres envoyées d'Europe par les premiers engagés dahoméens. Ce sont ces lettres qui se trouvent dans son dossier d'archives. Elles témoignent de la prise d'écriture de jeunes gens dont l'allégeance envers la France sert des ambitions individuelles d'élévation sociale.

Ange Da Costa, dont j'ai choisi ici l'une des lettres, est l'un des épistoliers les plus à l'aise, il écrit au gouverneur plusieurs très longues lettres. Avant son engagement, il fut commis expéditionnaire des secrétariats généraux en Côte d'Ivoire puis commis archiviste des travaux publics au Dahomey, et après la guerre il travaillera au Gouvernement Général à Dakar. Dans une des toutes premières lettres que nous avons de lui, écrite depuis Saint-Raphaël le 6 avril 1916, il se met en scène ayant obtenu, par sa seule force de persuasion, à ne pas être déclaré inapte au combat et à partir sur le front. Pour cela, il doit convaincre le médecin qui lui fait passer une visite médicale, pour la $3^{\text {ème }}$ fois :

Le même médecin-major de 1re cl. rouge de colère me toisa en disant: "Figurez-vous que je ne connais pas mon métier et que ce soit vous qui puissiez me contredire ? " Comme transfiguré je ne savais même pas d'où me venaient les mots que je plaidais ma cause tout en lui analysant mon indignation de me voir porter comme inapte à faire campagne. Non seulement j'ai remporté sur lui une victoire inattendue mais je fus félicité par lui en me serrant la main. La dernière parole pour moi fut : " Votre couleur cache à plusieurs gens une âme vraiment française ". II me renvoya ensuite en portant sur mon billet de visite : « apte à faire campagne sur sa demande. ${ }^{9}$

Le récit ici inséré dans la lettre a une portée argumentative évidente. Servant à l'auto-valorisation du narrateur, il met en scène une interaction inégale dans un rapport dominé-dominant (ici, le médecin de première classe français vs le tirailleur africain), où le dominé retourne la situation en sa faveur, par sa seule éloquence. La victoire est double : Da Costa obtient ce qu'il souhaite, à savoir être déclaré apte à aller au front, et il parvient à briser le préjugé racial, ce que confirme la citation en style direct attribué au médecin : « Votre couleur cache à plusieurs gens une âme vraiment française ». Ce récit, adressé dans une lettre au gouverneur du Dahomey, sert aussi une stratégie à plus long terme, celle de la reconnaissance de ses droits à la citoyenneté française.

À travers cet exemple se dessinent les contours d'une voix conçue comme voix qui porte, où sont en jeu à la fois une compétence de communication au sens de Dell Hymes (1996), et la capacité de parler «à 
Van den Avenne, Cécile. "La voix des « indigènes », ou comment rendre audible des voix rendues muettes. Sociolinguistique dans les archives coloniales. » Nouvelle Revue Synergies Canada, №15

propos » comme dirait Bourdieu (1977, p.18), de maîtriser les codes de la communication efficace dans un contexte social défini.

La question dès lors qu'on pourrait se poser en ce point du parcours serait : faut-il user de la langue dominante pour se construire une voix qui porte? L'efficience doit-elle passer par une forme de perte, celle de la langue, des langues premières, locales? On peut se demander aussi s'il n'y a pas un effet de sources: le chercheur travaillant sur les fonds d'archives disponibles, et j'en ferais alors partie, participeraient d'une forme d'invisibilisation de voix qui se seraient exprimées dans d'autres langues que les langues européennes, qu'il n'entend pas, tout à la fois parce qu'il n'investigue pas d'autres fonds que les fonds disponibles, et qu'il n'entend pas parce qu'il ne comprend pas ces langues.

\section{Ouverture : Des voix en langues africaines - Des archives alternatives}

J'évoquerai donc brièvement pour finir un projet, auquel je participe, mené par l'historienne Camille Lefebvre $^{10}$, spécialiste du Niger à époque pré-coloniale et coloniale, et qui travaille avec une équipe à rassembler des sources écrites en haussa, la langue véhiculaire dominante dans cette partie de l'Afrique, sources produites avant l'arrivée des colons européens, ou qui attestent d'usage de cette langue entre Africains et Européens.

Cela permet de recueillir des récits alternatifs aux récits coloniaux dominants en langue européenne, mais cela permet aussi de mettre au jour des transactions linguistiques entre Européens et Africains qui ne passent pas par les langues européennes, et qui suivent des conventions de communication propres à cette partie de l'Afrique. Cela permet aussi enfin de rendre compte de pratiques locales de la littératie, inscrites dans un contexte social qu'il s'agit de comprendre. Ou l'agentivité est aussi une agentivité locale, qui échappe à la domination coloniale, et de fait l'ensemble des enjeux locaux de pouvoir et de savoir ne peut pas être appréhendé au filtre de la relation coloniale.

Pour conclure, ou plutôt ouvrir la discussion, j'espère tout d'abord avoir montré la spécificité de mon approche de la notion de voix, croisant histoire et sciences sociales du langage, la question des effets de source et la façon de les prendre en compte sérieusement, en lisant les archives " along the grain » ou « against the grain » comme le préconise notamment l'historienne Ann Stoler (2019[2009]), pour en comprendre la logique propre d'une part, pour faire surgir des voix effacées, recouvertes d'autre part. En outre, j'espère avoir aussi montré l'épaisseur conceptuelle de la notion de voix, où les dimensions proprement langagières et linguistiques (répertoires linguistiques et langagiers, compétences de communication) ne peuvent être dissociés d'usages situés dont il faut comprendre les logiques et les enjeux.

\section{Notes}

1 Cette première partie de cadrage reprend des éléments de mon mémoire d'habilitation à diriger des recherches, non publié (Van den Avenne, 2015).

${ }^{2}$ Ma traduction.

3 J'emprunte à Michael Silverstein et Greg Urban la notion d'entextualisation désignant un procédé par lequel un énoncé discursif devient un texte, à partir du moment où il est détachable de son contexte d'énonciation.

${ }^{4}$ Sur la violence inhérente aux procédés de traduction, notamment dans ses liens à l'histoire coloniale, je renvoie à l'essai récent de Tiphaine Samoyault (2020).

${ }^{5}$ À Sedd-Ul Bhar eut lieu, du 25 avril au 4 mai 1915, l'une des batailles sanglantes de l'expédition des Dardanelles, au cours de laquelle les troupes franco-britanniques débarquèrent dans la péninsule de Gallipoli.

${ }^{6}$ Dans les archives militaires, les noms des tirailleurs sont presque systématiquement déformés. Sous le matricule 16568 et ce nom ridiculement transcrit, il s'agit sans doute de Sory (prénom) Coulibaly (patronyme). Il était né à Kati, cultivateur, et marié. 
Van den Avenne, Cécile. "La voix des « indigènes », ou comment rendre audible des voix rendues muettes. Sociolinguistique dans les archives coloniales. » Nouvelle Revue Synergies Canada, №15

7 L'intégralité du dossier du conseil de guerre est consultable en ligne, sur la base nominative des fusillés de la Première guerre mondiale, dossier SHD/GR $11 \quad \mathrm{~J} \quad 3184$, http://memoiredeshommes.sga.defense.gouv.fr

${ }^{8}$ Pour une description précise de la politique linguistique menée au sein de l'armée coloniale, je renvoie à mon article, Van den Avenne, 2005.

${ }^{9}$ Archives Nationales d'Outre Mer, Fonds Ministériel, EE/II/1161bis.

${ }^{10}$ LANGARCHIV, Language as archive: European linguistics and the social history of the Sahara and Sahel in the eighteenth and nineteenth century, ERC Starting Grant 2017. 
Van den Avenne, Cécile. "La voix des « indigènes », ou comment rendre audible des voix rendues muettes. Sociolinguistique dans les archives coloniales. » Nouvelle Revue Synergies Canada, №15

\section{Bibliographie}

Bertrand, Romain. L'histoire à parts égales, Paris, Seuil, 2011.

Binger, L.-G. (1892). Du Niger au golfe de Guinée. Paris, Hachette.

Blommaert, J. (2004). Writing as a problem: African grassroots writing, economies of literacy, and Globalization. Language in Society, 33(5), 643-671.

Blommaert, J. (2008a). Grassroots literacy: Writing, identity and voice in Central Africa. Routledge.

Blommaert, J. (2008b). Bernstein and poetics revisited: Voice, globalization and education. Discourse and Society, 19(4), 425-451.

Bourdieu, P.. (1977) L'économie des échanges linguistiques. Langue française, 34, 17-34.

Branca-Rosoff, S. et Schneider, N. (1994). L'écriture des citoyens. Une analyse linguistique de l'écriture des peu-lettrés pendant la période révolutionnaire. Pairs Klincksieck.

Branca-Rosoff, S. (1989). Vue d'en bas: Des écrits «malhabiles » pendant la période révolutionnaire. Langage et société, 47(1), 9-27.

Certeau, M. (1990). L'invention du quotidien, t.1. Arts de faire, Gallimard.

Certeau, M. (1975). L'écriture de l'histoire. Gallimard.

Chaudenson, R. (2003). La créolisation : Théorie, applications, implications. L'Harmattan.

Clément, A. (2014). À la recherche des "voix " des fallāhīn dans un dossier d'archives judiciaires égyptiennes. Ateliers d'anthropologie [Online] 36, consulté le 4 février 2014. URL: http://ateliers.revues.org/9007 ; DOI : 10.4000/ateliers.9007.

Duranti, A. (dir.). (2007). A Companion to Linguistic Anthropology. Blackwell Publishing, 2007(2004).

Fabre, D. (1993). Écritures ordinaires. P.O.L./ Centre Georges Pompidou /B.P.I.

Hirschman, A. O. (1995) Défection et prise de parole : théorie et applications, Fayard.

Hymes, D. H. (1996). Ethnography, linguistics, narrative inequality: Toward an understanding of voice. Taylor \& Francis.

Keane, W. (2007). Voice. Dans A. Duranti (dir.), Key Terms in Language and Culture. Blackwell Publishing, 268-271.

Payet, J.-P., Giuliani, F. et Laforgue, D. (dir.). (2008). La voix des acteurs faibles. De l'indignité à la reconnaissance. Presses universitaires de Rennes, collection Le sens social.

Petrucci, A. (1986). Strittura e Popolo. Piccola Biblioteca Inaudi.

Samoyault, T. (2020). Traduction et violence. Seuil.

Silverstein, Mi. et Urban, G. (1996). Natural Histories of Discourse. University of Chicago Press.

Spivak, G. C.(1988). Can the Subaltern Speak? Dans C. Nelson et L. Grossberg (dir.), Marxism and the Interpretation of Culture (p. 271-313). University of Illinois Press. (Les Subalternes peuvent-illes parler? Traduction française de Jérôme Vidal, Éditions Amsterdam, 2006).

Stoler, A. (2019 [2009]) Au cœur de l'archive coloniale. Questions de méthode, Paris, EHESS.

Tharaud J. et Tharaud J., (1922) La randonnée de Samba Diouf, Paris, Plon.

Van den Avenne, C. (2019). La fabrication textuelle du «français africain » : Entextualisation, mises en scènes, réceptions. Langue française, 202(2), 61-75. 
Van den Avenne, Cécile. "La voix des « indigènes », ou comment rendre audible des voix rendues muettes. Sociolinguistique dans les archives coloniales. » Nouvelle Revue Synergies Canada, N¹5

Van den Avenne, C. (2017a). L'exploration coloniale en ses langues. Une philologie des carnets africains du capitaine Binger. Genèses. Sciences sociales et histoire, 106, 131-153.

Van den Avenne, C. (2017b). De la bouche même des Indigènes. Éditions Vendémiaire.

Van den Avenne, C. (2005). Bambara et français-tirailleur. Une analyse de la politique linguistique de l'armée coloniale française: la Grande Guerre et après. Documents pour l'histoire du français langue étrangère ou seconde SIHFLES, décembre 2005, 35, 123-150. 